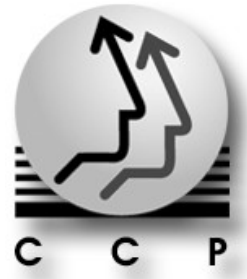

Población y Salud en Mesoamérica

Revista electrónica publicada por el

Centro Centroamericano de Población,

Universidad de Costa Rica, 2060 San José, Costa Rica

http://ccp.ucr.ac.cr

Población y Salud en Mesoamérica

Revista electrónica semestral, ISSN-1659-0201

Volumen 10, número 1, artículo 3

Julio - diciembre, 2012

Publicado 1 de julio, 2012

http://ccp.ucr.ac.cr/revista/

\title{
Evolución de la esperanza de vida de Costa Rica en el contexto global (1930-2010)
}

\section{Óscar Peláez-Herreros}

\section{(cc) EY-NC-ND}

Protegido bajo licencia Creative Commons

Centro Centroamericano de Población 


\title{
Evolución de la esperanza de vida de Costa Rica en el contexto global (1930- 2010)
}

\author{
Evolution of life expectancy in Costa Rica in global context (1930-2010)
}

\section{RESUMEN}

Óscar Peláez-Herreros ${ }^{1}$

Esta investigación analiza la evolución de la esperanza de vida al nacimiento en Costa Rica en el marco de las dinámicas globales de esta variable con el objetivo de encontrar factores que ayuden a explicar sus fases de crecimiento acelerado, desaceleración y estancamiento. Para ello, en primer lugar, recurriendo a los conceptos de convergencia $\beta$ y $\sigma$, se confirma la existencia de un proceso generalizado de convergencia al alza de las esperanzas de vida de las distintas poblaciones del mundo. Posteriormente, se advierte que, hasta principios de la década de los ochenta, la serie de Costa Rica se aproximó a la de mejores prácticas a un ritmo superior a la media, para, a partir de entonces, ralentizar su crecimiento y evolucionar en paralelo a la serie de máximos. Tras integrar las explicaciones de la dinámica particular de la serie de Costa Rica en el marco general, donde el margen existente para incorporar innovaciones es relevante, se estudian los factores estructurales que pueden estar limitando la capacidad de ciertas poblaciones para adoptar las mejores prácticas vigentes en cada momento, impidiendo que su brecha respecto al máximo se cierre completamente, como es el caso de Costa Rica desde 1980. Para ello, se estima un modelo de datos de panel con dinámica de errores que ofrece evidencia de que el porcentaje del producto interno bruto destinado a gasto público en salud y, especialmente, el grado de urbanización son variables relevantes en este sentido.

Palabras clave: esperanza de vida, convergencia, difusión de innovaciones, gastos públicos, urbanización

\begin{abstract}
This research analyzes the evolution of life expectancy at birth in Costa Rica under the global dynamics of this variable in order to find factors that help to explain its stages of rapid growth, deceleration and stagnation. To do this, first, using the concepts of $\beta$-convergence and $\sigma$-convergence, the existence of a generalized process of upward convergence of life expectancies is confirmed. Afterwards, it is noted that, until the early eighties, the series of Costa Rica approached to best practices at a higher pace than average. Thereafter, the growth of the series slows, beginning to move in parallel with this of best practices. After integrating the explanations of the particular dynamics of Costa Rica in the general framework, where the innovations gap is relevant, the structural factors that may be limiting the ability of certain populations to adopt best practices, as in the case of Costa Rica since 1980, are analyzed. With this purpose, we estimate a dynamic panel data model that provides evidence that the percentage of gross domestic product engaged to public health and, especially, the degree of urbanization are significant variables in this regard.
\end{abstract}

Keywords: life expectancy, convergence, diffusion of innovations, public expenditures, urbanization.

Recibido: 13 ene. 2012

Aprobado: 5 mar. 2012

\footnotetext{
${ }^{1}$ El Colegio de la Frontera Norte, Departamento de Estudios Económicos. MEXICO. opelaez@colef.mx
} 


\section{INTRODUCCIÓN}

En el régimen demográfico antiguo, la muerte era un fenómeno habitual que impedía que un tercio de los nacidos alcanzara la edad adulta. La falta de higiene, la escasez de alimentos en los años de mala cosecha o el desconocimiento de las causas y del tratamiento de las enfermedades hacía que cada año falleciera entre un tres y un cuatro por ciento de la población (Vallin, 1995), una cifra muy superior al uno por ciento que se observa en la actualidad tanto en los países desarrollados como en muchos en desarrollo.

A diferencia de otros indicadores relacionados con la capacidad de supervivencia de las poblaciones, como el número de fallecidos o la tasa de mortalidad, la esperanza de vida al nacimiento no depende del tamaño ni de la composición por edades del colectivo al que representa. Esto supone una ventaja importante ya que permite la comparación de valores, no sólo a lo largo del tiempo, sino también entre poblaciones diferentes.

En Costa Rica, la esperanza de vida prácticamente se ha duplicado en las últimas ocho décadas, pasando de los 42,23 años registrados en 1930 a los 79,04 en 2010 (CCP, 2011). Los progresos en saneamiento básico, salud pública, atención hospitalaria, alimentación y educación han contribuido a este destacado avance (Horwitz, 1987; Rosero-Bixby, 1991 y 1994; Robles, 2006) que, en cualquier caso, no ha mantenido un ritmo constante a lo largo de todo el periodo referido.

En la década de los noventa, por ejemplo, la esperanza de vida apenas aumentó desde los 77,00 años observados en 1990 a los 77,74 de 2000. En décadas previas, los ritmos de crecimiento fueron mucho mayores, como en la parte central del siglo, cuando la esperanza de vida de la población costarricense se incrementó a razón de casi un año por cada año de calendario, pasando de los 46,93 de 1940 a los 65,66 de $1960 .^{2}$ La década de los sesenta, sin embargo, también presentó una evolución muy pobre. En 1968, la serie alcanzó un máximo relativo de 67,79 años, para retroceder hasta los 65,86 en 1970, un valor ligeramente superior al de 1960. Como comenta Rosero-Bixby (1991), esta dinámica encuentra explicación en los efectos que tuvieron las erupciones de los volcanes Irazú, en 1963-1965, y Arenal, a partir de 1968.

La década de los setenta puede considerarse como el último periodo de rápido crecimiento de la esperanza de vida en Costa Rica, que alcanzó los 74,62 años en 1980, aumentando diez meses por cada año transcurrido. A partir de entonces, la expansión de la serie volvió a ralentizarse, llegando al estancamiento, ya mencionado, durante los noventa. Factores relacionados con la reducción de la mortalidad infantil y juvenil (Haines y Avery, 1982; Rosero-Bixby, 1986) ayudan a explicar este último periodo de rápido crecimiento de la esperanza de vida.

Además de los motivos particulares, que no conviene olvidar, la posibilidad de mejorar la capacidad de supervivencia de cualquier población está limitada por los avances que se producen a nivel mundial en los conocimientos o en el tratamiento de enfermedades. En 1950, por ejemplo, una esperanza de vida al nacimiento de 79 años, como la actual de Costa Rica, no sólo era

\footnotetext{
${ }^{2}$ Este periodo coincide con el que Camposortega (1992:384) califica como de "disminución extraordinaria" de la mortalidad en México. Según estimaciones de Camposortega (1992), la capacidad media de supervivencia de la población mexicana femenina aumentó desde los 41,65 años de 1940 a los 59,73 de 1960. Para los hombres, el avance fue en paralelo desde los 39,12 años a los 55,99. Cifras similares presentan Benítez y Cabrera (1967), Arriaga (1968) y Corona et al. (1982).
} 
inalcanzable, sino incluso difícil de imaginar. Por aquel entonces, en los países más avanzados, la incidencia de las enfermedades infecciosas y parasitarias ya se había reducido, ganando relevancia las crónicas y degenerativas. La transición epidemiológica parecía estar completa (Omran, 1971) y el límite biológico de la especie humana, que Bourgeois-Pichat (1952) había cifrado en 76,3 años de esperanza de vida para los hombres y en 78,2 para las mujeres, se mostraba insuperable. La esperanza de vida más alta era la de Noruega $(71,60)$, Países Bajos $(71,45)$ y Suecia $(71,14){ }^{3}$

Con el transcurso del tiempo, el progresivo avance en el tratamiento de las enfermedades crónicas permitió que estas poblaciones transitaran desde "la edad de las enfermedades degenerativas y causadas por el hombre" (Omran, 1971) a "la edad de las enfermedades degenerativas diferidas" (Olshansky y Ault, 1986). En la actualidad, por ejemplo, la esperanza de vida de la población noruega se eleva hasta los 80,98 años, mientras que la japonesa llega hasta los 83,31, superando ampliamente los límites calculados por Bourgeois-Pichat (1952), Whelpton et al. (1947) o Frejka (1981), entre otros (Manton et al., 1991; Oeppen y Vaupel, 2002). Asimismo, la difusión y asimilación de hábitos más saludables y de los avances generados en las poblaciones más avanzadas en el combate a la mortalidad, lo que Rosero-Bixby (1991) denomina "importación de tecnologías de salud de bajo costo y alta eficacia", ha permitido que la mayoría de poblaciones rezagadas en este aspecto recorten la distancia que mantenían con las primeras (Davis, 1956), dando lugar a la convergencia global detectada por Wilson (2001) y Bongaarts (2006).

Debido a la interrelación creciente que permite la transmisión de progresos entre poblaciones, es conveniente valorar la dinámica de la esperanza de vida de un país, o de una región concreta, no como una tendencia aislada, sino inmersa en el contexto global, ya que es dependiente de las posibilidades que en éste existen en cada momento, entendiendo que éxitos y fracasos son relativos. Por este motivo, en el presente artículo, se pretende analizar la evolución a largo plazo de la esperanza de vida de Costa Rica en comparación con otros países y territorios del mundo, complementando, así, las explicaciones dadas por otros autores, y poniendo especial énfasis en los factores estructurales diferenciales que ayudan a explicar el pobre desempeño de la serie desde principios de la década de los ochenta.

Para ello, en primer lugar, se describe la evolución que la esperanza de vida al nacimiento ha tenido en distintas poblaciones, presentando los métodos que permiten el análisis conjunto y comparado de estas series, así como los datos a los que se recurre. Posteriormente, se analizan los resultados de la investigación, tratando de ver cómo fue la evolución de Costa Rica en el contexto global, esto es, si el país aprovechó las condiciones existentes a partir de la experiencia de otras poblaciones o, en su caso, cuáles fueron las causas que lo impidieron. Por último, se establecen las conclusiones más relevantes de este estudio.

\footnotetext{
${ }^{3}$ Datos extraídos de la Human Mortality Database (HMD, 2011).
} 


\section{PLANTEAMIENTO DEL PROBLEMA, DATOS Y MÉTODOS}

Hasta hace pocos siglos, la esperanza de vida de la especie humana permaneció estable a largo plazo entre los 20 y los 35 años de edad. Desde las investigaciones que la sitúan en los 21 años durante el paleolítico (Vallin, 1995) o los 22 años para las mujeres y los 25 para los hombres durante los siglos II a IV d.C. en el antiguo Egipto (Bagnall, 1993:182), hasta los 25 años que Livi-Bacci (1978:181 y 2002:147) fija para la población francesa de mediados del siglo XVIII o los 32 que Spengler (1978:104) estima para Inglaterra en 1690, apenas existen variaciones en los valores observados para esta variable. Según Arriaga y Davis (1969:226), en América Latina, alrededor de 1900, la esperanza de vida oscilaba entre los 24 y los 30 años de edad. En Paraguay, por ejemplo, en 1883 aún era de 23 años, en Brasil en 1872 alcanzaba los 27, mientras que en Costa Rica en 1864 llegaba a los 26. Pérez-Brignoli (2010) sugiere valores de esperanza de vida ligeramente superiores a éstos, que en promedio rondaban los 30 años pero, en cualquier caso, comprendidos entre los 20 y los 40 años de edad.

La capacidad de supervivencia de las poblaciones sólo comenzó a aumentar de forma sostenida tras las revoluciones agrícola e industrial que tuvieron origen en Inglaterra en los siglos XVII y XVIII. Las mejoras en la higiene y en la nutrición evitaron un buen número de fallecimientos, sobre todo, entre la población más joven. Desde entonces, la esperanza de vida de aquel país ha ido elevándose hasta los 46,32 años en 1900, los 69,03 en 1950 y los 80,57 en 2009. Los cambios iniciados en Inglaterra pronto se extendieron a otros países, aunque con calendarios e intensidades diferentes, primero dentro del continente europeo, pero luego también fuera de él. ${ }^{4}$

Esta expansión progresiva dio origen a una divergencia de las series de esperanza de vida de las distintas poblaciones a nivel mundial. Aquéllas que disfrutaron de los cambios iniciales comenzaron a distanciarse de la mayoría, que aún permanecía bajo las condiciones propias del régimen demográfico antiguo. No obstante, a medida que las mejores prácticas relacionadas con la supervivencia se fueron difundiendo, un mayor número de poblaciones logró que su esperanza de vida abandonara el estancamiento histórico en que se encontraba, ${ }^{5}$ alcanzando ritmos de crecimiento superiores a los de los países pioneros, dado que éstos tuvieron que soportar los costos y tiempos de innovación (Davis, 1956; Arriaga y Davis, 1969:227-31). El desarrollo de las comunicaciones y los medios de transporte ayudó a la reducción de las diferencias existentes en cuanto a modos de vida, aplicación de políticas de salud pública, acceso a antibióticos, vacunas, insecticidas, alimentación más variada, etcétera (Davis, 1956; Riley, 2001), favoreciendo el acercamiento de los seguidores con independencia de su menor grado de desarrollo económico, lo que dio lugar al proceso de convergencia que Wilson (2001) y Bongaarts (2006) detectan durante la segunda mitad del siglo XX.

\footnotetext{
${ }^{4}$ Arriaga y Davis (1969:226-7) comentan que, en el caso de América Latina, la reducción significativa de la mortalidad se inició después del año 1910, haciéndose más notable a partir de 1930. Pérez-Brignoli (2010:13-4) señala que en Costa Rica, Chile, Brasil, México, Venezuela, Colombia, Panamá y Paraguay la esperanza de vida al nacimiento superó los cuarenta años durante la década de 1930. En Argentina, Uruguay y Cuba esta cota se rebasó incluso antes de la Primera Guerra Mundial, mientras que los restantes países de América Latina tuvieron que esperar hasta las décadas de 1940 y 1950 para lograrlo.

${ }^{5}$ El estancamiento al que se hace referencia es el de la tendencia de la serie a largo plazo, ya que, a corto plazo, la variabilidad de la esperanza de vida era mayor en épocas precedentes que en la actualidad debido a que algunos acontecimientos, como las malas cosechas o las epidemias, tenían gran incidencia en la mortalidad registrada en ciertos años.
} 
Al analizar la esperanza de vida al nacimiento en Costa Rica junto con las dinámicas globales de esta variable, se pretende conocer con detalle la cronología de su evolución, buscando factores que ayuden a explicar sus fases de crecimiento acelerado, así como de estancamiento, dentro del marco de divergencia-convergencia que se acaba de esbozar. Para ello, en primer lugar, se estudia este proceso mediante una metodología semejante a la descrita por Barro y Sala-i-Martin (1990, 1991, 1992 y 1995). Ello permitirá comprobar si la esperanza de vida creció más en las poblaciones donde presentaba valores más bajos al inicio del periodo de análisis (convergencia $\beta$ ) $\mathrm{y}$, también, si para el conjunto de poblaciones estudiadas, se redujo, o no, la dispersión de los niveles de esperanza de vida (convergencia $\sigma$ ).

Para realizar este análisis, se recurre a la información publicada en la base de datos del Banco Mundial (BM, 2011), que aglutina series de esperanza de vida para 212 poblaciones con periodicidad anual, prácticamente completas a lo largo del periodo 1960-2009. Teniendo en cuenta que la esperanza de vida no arroja resultados robustos a lo largo del tiempo para poblaciones poco numerosas y que el método de análisis que se pretende utilizar no distingue entre tamaños de poblaciones, con el fin de evitar que distorsiones de este tipo alteren las tendencias generales que se quieren develar, a semejanza de la investigación realizada por Chenery y Syrquin (1975), se prescinde de los países y territorios que en 2009 no alcanzaban el millón de habitantes. Asimismo, no se consideran los datos correspondientes a Serbia, Kosovo y Ribera Occidental y Gaza, para los que únicamente hay información disponible a partir de fechas recientes. De este modo, el número de series a considerar se reduce a $152,{ }^{6}$ representando a 6.723 de los 6.775 millones de habitantes que el planeta tenía en 2009 según BM (2011).

Teniendo en cuenta la limitación temporal impuesta por la disponibilidad de información, el método que se utiliza para comprobar si en el periodo 1960-2009 hubo convergencia $\beta$ entre las esperanzas de vida de las principales poblaciones, consiste en ajustar un modelo lineal del tipo:

$$
\Delta \mathrm{e}_{\mathrm{i}, 2009}=\alpha+\beta \cdot e_{\mathrm{i}, 1960}+\varepsilon_{i}
$$

donde $\Delta \mathrm{e}_{\mathrm{i}, 2009}=\mathrm{e}_{\mathrm{i}, 2009}-\mathrm{e}_{\mathrm{i}, 1960}$ es la variación de la esperanza de vida al nacimiento en la población i-ésima entre los años 1960 y 2009; $\alpha$ y $\beta$ son los parámetros del modelo; y $\varepsilon_{\mathrm{i}}$ es el término de error. $^{7}$ Dada esta expresión, un valor negativo y estadísticamente significativo del parámetro $\beta$ indica la existencia de una relación inversa entre los valores iniciales de esperanza de vida, $\mathrm{e}_{\mathrm{i}, 1960}$,

${ }^{6}$ Los 152 países y territorios considerados son: Afganistán, Albania, Alemania, Angola, Arabia Saudita, Argelia, Argentina, Armenia, Australia, Austria, Azerbaiyán, Bangladesh, Bélgica, Benin, Bielorrusia, Bolivia, Bosnia y Herzegovina, Botswana, Brasil, Bulgaria, Burkina Faso, Burundi, Camboya, Camerún, Canadá, Chad, Chile, China, Colombia, Costa de Marfil, Costa Rica, Croacia, Cuba, Dinamarca, Ecuador, Egipto, El Salvador, Emiratos Árabes Unidos, Eritrea, Eslovaquia, Eslovenia, España, Estados Unidos, Estonia, Etiopía, Filipinas, Finlandia, Francia, Gabón, Gambia, Georgia, Ghana, Grecia, Guatemala, Guinea, Guinea-Bissau, Haití, Honduras, Hong Kong, Hungría, India, Indonesia, Irán, Iraq, Irlanda, Israel, Italia, Jamaica, Japón, Jordania, Kazajstán, Kenya, Kirguistán, Kuwait, Lesotho, Letonia, Líbano, Liberia, Libia, Lituania, Macedonia, Madagascar, Malasia, Malawi, Malí, Marruecos, Mauricio, Mauritania, México, Moldavia, Mongolia, Mozambique, Myanmar, Namibia, Nepal, Nicaragua, Níger, Nigeria, Noruega, Nueva Zelanda, Omán, Países Bajos, Pakistán, Panamá, Papua Nueva Guinea, Paraguay, Perú, Polonia, Portugal, Puerto Rico, Qatar, Reino Unido, República Centroafricana, República Checa, República de Corea, República Democrática del Congo, República Democrática Popular Lao, República del Congo, República Dominicana, República Popular Democrática de Corea, Rumanía, Rusia, Rwanda, Senegal, Sierra Leona, Singapur, Siria, Somalia, Sri Lanka, Sudáfrica, Sudán, Suecia, Suiza, Swazilandia, Tailandia, Tanzania, Tayikistán, Timor-Leste, Togo, Trinidad y Tobago, Túnez, Turkmenistán, Turquía, Ucrania, Uganda, Uruguay, Uzbekistán, Venezuela, Viet Nam, Yemen, Zambia y Zimbabwe. 
y la variación experimentada por esta variable durante el periodo considerado, $\Delta \mathrm{e}_{\mathrm{i}, 2009}$. En ese caso, la esperanza de vida al nacer habría crecido más en aquellas poblaciones donde era menor en 1960 (convergencia $\beta$ ).

Por otra parte, para el conjunto de poblaciones comentado, se considera que existe convergencia $\sigma$ si la dispersión de sus esperanzas de vida se reduce con el paso del tiempo, esto es, si $\sigma_{2009}<\sigma_{1960}$ o, lo que es lo mismo, si la desviación típica para el año 2009 es menor que en 1960.

Una vez que se confirme o refute el proceso de convergencia a nivel mundial y, en su caso, se conozca la intensidad con que las poblaciones con esperanzas de vida más bajas recuperaron el terreno perdido respecto a las más avanzadas, se analiza el caso particular de Costa Rica, buscando las causas concretas de su dinámica. ${ }^{8}$ Para ello, se estudia su evolución en comparación con otras poblaciones, prestando especial atención a las del área centroamericana, con las que, por ejemplo, comparte condiciones climatológicas, epidemiológicas y culturales.

Asimismo, se analiza en qué medida se fue reduciendo o ampliando su distancia respecto a la serie de esperanza de vida máxima a nivel mundial consecuencia de la aplicación de las mejores prácticas existentes en cada momento (Oeppen y Vaupel, 2002). Para construir esta serie, se recurre a los datos de esperanza de vida de BM (2011), pero también a los publicados en la Human Mortality Database (HMD, 2011), ${ }^{9}$ prácticamente idénticos a los de BM (2011) para los años y poblaciones en que ambas bases coinciden. ${ }^{10}$ La ventaja de los datos de HMD (2011) es que permiten extender el periodo de estudio hacia el pasado. El inconveniente es que sólo recogen información para 40 poblaciones, lo que impide realizar el análisis de convergencia anterior, motivo por el cual, en ese caso, se recurre exclusivamente a las series de BM (2011).

\footnotetext{
${ }^{7}$ A diferencia del planteamiento de Barro y Sala-i-Martin (1990, 1991, 1992 y 1995), en este caso no se recurre a tasas acumulativas de variación, ya que la esperanza de vida no evoluciona en forma acumulativa, como sí lo hace el producto interno bruto per cápita, sino en forma, a lo sumo, constante (Oeppen y Vaupel, 2002) con independencia del nivel.

${ }^{8}$ La serie de esperanza de vida al nacimiento de Costa Rica se obtuvo del sitio web del Centro Centroamericano de Población (CCP, 2011). Está formada por observaciones para los años 1930, 1940 y 1950-2010. Al compararla con los datos correspondientes de BM (2011), que abarcan el lapso 1960-2009, se observan algunas diferencias, especialmente, a principios de los sesenta, en la segunda mitad de los setenta y durante la década de los ochenta. Estas diferencias, de alrededor de uno o dos años, se explican por el hecho de que la serie de BM (2011) está suavizada. Por ello, se decide utilizar la de CCP (2011), ya que permite un análisis más detallado de la dinámica de la esperanza de vida de Costa Rica. En cualquier caso, la tendencia de ambas series es muy parecida y los valores son casi coincidentes a partir de 1990. HMD (2011) no incluye información sobre Costa Rica.

${ }^{9}$ La construcción de la serie de esperanza de vida máxima a nivel mundial con los datos de BM (2011) se realiza con las 152 poblaciones señaladas anteriormente. En el caso de HMD (2011), se utilizan los datos correspondientes a las siguientes poblaciones y periodos temporales: Alemania Occidental (1956-2009), Alemania Oriental (1956-2009), Australia (1921-2007), Austria (1947-2008), Bélgica (1841-2009), Bielorrusia (1959-2010), Bulgaria (1947-2009), Canadá (1921-2007), Chile (1992-2005), Dinamarca (1835-2009), Escocia (1855-2009), Eslovaquia (1950-2009), Eslovenia (1983-2009), España (1908-2009), Estados Unidos (1933-2007), Estonia (1959-2009), Finlandia (18782009), Francia (1816-2009), Hungría (1950-2009), Inglaterra y Gales (1841-2009), Irlanda (1950-2009), Irlanda del Norte (1922-2009), Islandia (1838-2008), Israel (1983-2009), Italia (1872-2008), Japón (1947-2009), Letonia (19592009), Lituania (1959-2009), Luxemburgo (1960-2009), Noruega (1846-2009), Nueva Zelanda (1948-2008), Países Bajos (1850-2009), Polonia (1958-2009), Portugal (1940-2009), República Checa (1950-2009), Rusia (1959-2009), Suecia (1751-2010), Suiza (1876-2009), Taiwán (1970-2009) y Ucrania (1959-2008).

${ }^{10}$ En la mayoría de los casos, las diferencias entre BM (2011) y HMD (2011) son inferiores al medio año. El año de discrepancia sólo se supera en los datos de Bielorrusia (1960-78, 1981-2 y 1987), Estonia (1964-5 y 1967), Israel (1988), Portugal (1960, 1965 y 1973-4), Rusia (1960-1, 1964 y 1992) y Ucrania (1960-1, 1964, 1966, 1971-8 y 1986).
} 
Al analizar el proceso de convergencia de la esperanza de vida de Costa Rica, se encuentra, como se comenta en detalle en el próximo apartado, que ésta se aproxima rápidamente a la de mejores prácticas hasta principios de la década de los ochenta. A partir de entonces, tiende a evolucionar en paralelo con respecto a la serie de Japón, el líder mundial en este aspecto. A la vista de que esta dinámica no es exclusiva de la esperanza de vida de Costa Rica, sino que es común a otras poblaciones, se estudia la existencia de factores estructurales que pueden estar limitando la capacidad de ciertas poblaciones para adoptar las mejores prácticas vigentes en cada momento, impidiendo que su brecha respecto al máximo se cierre completamente.

Para analizar estas tendencias, se identifican las poblaciones que presentan la evolución descrita, recurriendo a la información de BM (2011) dadas las características anteriormente comentadas de esta base de datos así como por el hecho de reunir información sobre variables adicionales que pueden ser relevantes a la hora de explicar este fenómeno. Procediendo de este modo, se encuentran 28 poblaciones en las que la esperanza de vida ha venido evolucionando en paralelo respecto a la de Japón, manteniéndose a una distancia de menos de cinco años durante las tres décadas más recientes. Por otra parte, atendiendo a los aspectos que han sido claves en la evolución de la serie de Costa Rica y a los que señalan estudios como los de Preston (1975), Rodgers (1979) o Anand y Ravallion (1993), se eligen las siguientes cuatro variables como posibles factores explicativos de las brechas mencionadas: ingreso nacional bruto per cápita en paridades de poder adquisitivo $\left(\mathrm{X}_{1}\right),{ }^{11}$ gasto público en salud como porcentaje del producto interno bruto $\left(\mathrm{X}_{2}\right)$, gasto público en educación como porcentaje del producto interno bruto $\left(\mathrm{X}_{3}\right), \mathrm{y}$ porcentaje de población urbana $\left(\mathrm{X}_{4}\right) .{ }^{12}$ Debido a algunas deficiencias en la información, el estudio se limita a los años comprendidos entre 1995 y 2009, así como a 24 de las 28 poblaciones referidas. ${ }^{13}$

Con el fin de estudiar si los valores de estas variables ayudan a explicar las distancias de las esperanzas de vida respecto a la de Japón, esto es, $\mathrm{y}_{\mathrm{it}}=\mathrm{e}_{0 \mathrm{it}}-\mathrm{e}_{0 \mathrm{Jt}}$, donde el subíndice $\mathrm{J}$ hace referencia a los datos de Japón, todas ellas se expresan en diferencias respecto a los valores correspondientes de las series niponas: $\mathrm{d}_{\mathrm{kit}}=\mathrm{x}_{\mathrm{kit}}-\mathrm{x}_{\mathrm{kJt}}$ con $\mathrm{k}=1, \ldots, 4 ; \mathrm{i}=1, \ldots, 24 ; \mathrm{t}=1995, \ldots, 2009$. Debido al hecho de estar trabajando con series temporales, la estimación del modelo de datos de panel consecuente:

$$
y_{i t}=\alpha_{i}+\beta^{\prime} D_{i t}+\varepsilon_{i t}
$$

\footnotetext{
${ }^{11}$ El ingreso nacional bruto per cápita se considera en términos logarítmicos dado el crecimiento acumulativo de esta variable.

${ }^{12}$ Por desgracia, no fue posible incorporar al análisis variables que midieran los niveles de pobreza o de desigualdad en el ingreso de las poblaciones, ya que las series disponibles presentaban grandes carencias de datos.

${ }^{13}$ De las 28 poblaciones cuyas esperanzas de vida al nacimiento han venido evolucionando en paralelo y próximas a la de Japón en las últimas décadas, se descarta a cuatro (Cuba, Hong Kong, Puerto Rico y Qatar) ante la ausencia de datos para alguna de las variables seleccionadas. Se trabaja, por ello, con las series de: Alemania, Australia, Austria, Bélgica, Canadá, Chile, Costa Rica, Dinamarca, España, Estados Unidos, Finlandia, Francia, Grecia, Irlanda, Israel, Italia, Noruega, Nueva Zelanda, Países Bajos, Portugal, Reino Unido, Singapur, Suecia y Suiza.
} 
presenta autocorrelación de los residuos. Por este motivo, se plantea un modelo de datos de panel con dinámica de errores (Bhargava et al., 1982), capaz de eludir el problema de la autocorrelación:

$\varepsilon_{i t}=\rho \varepsilon_{i t-1}+u_{i t}$

al tomar diferencias respecto a un momento anterior:

$$
y_{i t-1}=\alpha_{i}+\beta^{\prime} D_{i t-1}+\varepsilon_{i t-1}
$$

multiplicar por $\rho$ :

$$
\rho y_{i t-1}=\rho \alpha_{i}+\rho \beta^{\prime} D_{i t-1}+\rho \varepsilon_{i t-1}
$$

y restar la ecuación [2] de la [1]:

$$
y_{i t}-\rho y_{i t-1}=\alpha_{i}-\rho \alpha_{i}+\beta^{\prime} D_{i t}-\rho \beta^{\prime} D_{i t-1}+\varepsilon_{i t}-\rho \varepsilon_{i t-1}
$$

que, teniendo en cuenta que $\varepsilon_{i t}-\rho \varepsilon_{i t-1}=u_{i t}$, puede reescribirse como:

$$
y_{i t}-\rho y_{i t-1}=\alpha_{i}(1-\rho)+\beta^{\prime}\left(D_{i t}-\rho D_{i t-1}\right)+u_{i t}
$$

donde el término constante, $\alpha_{\mathrm{i}}$, se elimina al utilizar una matriz simétrica idempotente, dando lugar al modelo de variables transformadas:

$$
y_{i t}^{i}=\beta^{\prime} D_{i t}^{i}+u_{i t}
$$

que puede estimarse por mínimos cuadrados ordinarios, generando, ahora sí, estimaciones eficientes de los parámetros. Este modelo permite explicar las diferencias en esperanza de vida, $\mathrm{y}_{\mathrm{it}}$, a partir de las diferencias observadas en cada una de las variables que componen el vector $\mathrm{d}_{\text {kit, }}$, esto es, ingreso nacional bruto per cápita $\left(\mathrm{D}_{1}\right)$, gasto público en salud $\left(\mathrm{D}_{2}\right)$, gasto público en educación $\left(\mathrm{D}_{3}\right)$ y porcentaje de población urbana $\left(\mathrm{D}_{4}\right)$, en el caso del presente estudio.

\section{RESULTADOS}

\subsection{Sobre la convergencia global}

Aplicando la metodología anterior a las series publicadas por BM (2011), es posible verificar la existencia del proceso de convergencia al alza en términos de esperanza de vida descrito por Wilson (2001). Como se observa en la Tabla 1, para el periodo 1960-2009, se obtiene un coeficiente de regresión negativo y estadísticamente significativo que indica que, en términos generales, la esperanza de vida al nacimiento aumentó más en aquellas poblaciones en las que en 1960 era más baja. Concretamente, por cada año de rezago inicial, tendió a aumentar 0,32 años adicionales entre 1960 y 2009. Como también se observa en la Tabla 1, la intensidad de este 
proceso no fue constante a lo largo del periodo analizado. Si bien en las décadas de los sesenta, setenta y en el nuevo milenio el coeficiente de regresión es plenamente significativo, éste deja de serlo en los ochenta (sólo es significativo al 95\% de confianza) y, especialmente, en los noventa. ${ }^{14}$

Resultados similares se obtienen al estudiar la evolución de la dispersión de las series de esperanza de vida (Gráfica 1). A pesar de que el nivel de la variable en consideración aumenta con el transcurso del tiempo, su desviación típica se reduce desde los 12,41 años de 1960 a los 10,44 de 2009. Los periodos de mayor convergencia vuelven a ser las décadas de los sesenta y los setenta. A través de esta medida, se aprecia con mayor precisión que la interrupción de la convergencia, también detectada en la Tabla 1, tuvo lugar a partir de mediados de los ochenta, prologándose hasta los primeros años del siglo entrante. El motivo de esta interrupción fue el mal desempeño de los países del África no mediterránea, cuyas esperanzas de vida, que ya eran las más bajas del planeta, se estancaron o incluso retrocedieron durante las décadas señaladas a causa, principalmente, de la propagación del síndrome de inmunodeficiencia adquirida (PNUD, 2006:265-7). Excluyendo del análisis a estas poblaciones, ${ }^{15}$ las restantes continuaron con el proceso de convergencia incluso durante los ochenta y los noventa como se desprende de la información contenida en la Tabla 1 y en la Gráfica 1.

\subsection{El área de Centroamérica y el Caribe}

En el caso de Centroamérica, ${ }^{16}$ se observa un intenso acercamiento entre las series desde la segunda mitad de los ochenta hasta fechas recientes (Gráfica 1), no ocurriendo lo mismo durante las décadas de los sesenta y los setenta, cuando la dispersión permaneció prácticamente inalterada. Esta dinámica en el interior del área, que también se aprecia al analizar los resultados de la Tabla 1, responde al desigual comportamiento de los países que lo integran.

La Gráfica 2 devela la existencia de dos grupos de poblaciones bien definidos dentro de la región centroamericana. Por un lado, Belice, Costa Rica y Panamá se caracterizan por haber iniciado el periodo objeto de estudio con esperanzas de vida sensiblemente más altas que los otros cuatro países del área. Por ejemplo, en 1960, la expectativa de supervivencia de la población costarricense superaba en 15,39 años a la de Honduras y en 15,86 a la de Guatemala.

Hasta 1980, las series de los tres países que partieron con ventaja se fueron aproximando al máximo, marcado fundamentalmente por Suecia hasta 1974 y por Japón desde entonces hasta la actualidad. En las tres décadas más recientes, ni Belice, ni Costa Rica, ni Panamá parecen haber sido capaces de reducir la distancia a la que entonces quedaron de la serie de máximos.

\footnotetext{
${ }^{14}$ La temporalidad detectada en el proceso de convergencia mediante la metodología descrita se corresponde con la que Wilson (2001:166-7) obtiene a partir de observar la evolución de los porcentajes de población mundial que se encuentra en cada uno de los intervalos quinquenales que establece para la esperanza de vida.

${ }^{15}$ Para el análisis de este subconjunto de poblaciones se prescinde de todos los territorios del continente africano con la excepción de Argelia, Egipto, Libia, Marruecos y Túnez, cuyas esperanzas de vida muestran evoluciones muy favorables a diferencia del resto de África. Con ello, el número de poblaciones se reduce de 152 a 111.

${ }^{16}$ Para el estudio de la convergencia en el área centroamericana se tienen en cuenta los datos de BM (2011) sobre Belice, Costa Rica, El Salvador, Guatemala, Honduras, Nicaragua y Panamá.
} 
El Salvador, Guatemala, Honduras y Nicaragua, que en 1960 tenían niveles más bajos, también mejoraron sus expectativas de supervivencia durante los sesenta y los setenta, pero a un ritmo que no fue suficiente para recortar la ventaja de los otros tres países de la región, que todavía estaban mejorando sus esperanzas de vida de forma acelerada para aproximarse al máximo. Por ello, en estas décadas, apenas hubo convergencia en Centroamérica.

Unido a ello, el retroceso que experimentó la serie de El Salvador desde 1975, fruto de la crisis política y social por la que atravesó el país y que acabaría desembocando en la guerra civil de los años ochenta, casi provocó la divergencia de las series de la región durante los setenta como ilustra el coeficiente estadísticamente nulo de la Tabla 1. A partir de los ochenta, el crecimiento más lento de las series de Belice, Costa Rica y Panamá, ya próximas al máximo, favoreció el progresivo acercamiento de los restantes países del área, que aún disponían de un margen de mejora amplio.

Al comparar la evolución de la esperanza de vida al nacimiento de Costa Rica con las series propias de las principales poblaciones del Caribe (Gráfica 3), se observan incluso más semejanzas que en relación a los países de Centroamérica (Gráfica 2). ${ }^{17}$ A excepción de Haití y República Dominicana, que presentan valores de esperanza de vida sensiblemente inferiores a los de Costa Rica; tanto Cuba, como Puerto Rico y, en menor medida, Jamaica y Trinidad y Tobago, muestran niveles y tendencias parecidos a los del país centroamericano. Destaca el hecho de que, si bien en 1960 la esperanza de vida de Costa Rica aún era inferior a la de estos países, ya en 1985 había superado sucesivamente a Trinidad y Tobago, Jamaica, Cuba y Puerto Rico, para situarse a la cabeza de todos ellos. En las tres décadas más recientes, sólo Puerto Rico y Cuba parecen mostrar una evolución similar a la de Costa Rica, ya que Jamaica y Trinidad y Tobago no han tenido tan buen desempeño, situándose a una distancia de casi diez años de la serie de mejores prácticas.

\subsection{El caso de Costa Rica}

La evolución de la serie de Costa Rica destaca, no sólo dentro del área centroamericana y del Caribe, donde ha sido un referente a lo largo del último medio siglo, sino también a nivel mundial. Si bien las distintas poblaciones han tendido a recuperar el terreno perdido respecto a las más avanzadas en esperanza de vida de forma acelerada, especialmente a partir del fin de la Segunda Guerra Mundial, la evolución de la serie de Costa Rica ha superado la dinámica media. Según datos de BM (2011), en el año 1960, la expectativa de vida de los costarricenses era la 50 ${ }^{a}$ más alta de las 152 que se consideran en este estudio. En 1975, ya ocupaba la posición 37, mejorando hasta la 23 en 1980 y hasta la 16 en 1985, posición que mantuvo durante los noventa, pero que ha cedido en la última década a favor de países como Corea del Sur, Eslovenia, Irlanda o Singapur. En 2009, Costa Rica era la 23ª población con esperanza de vida más alta.

Esta misma evolución puede observarse al analizar la distancia que la serie costarricense ha mantenido respecto al máximo. En este caso, se recurre a los datos de HMD (2011), que se

\footnotetext{
${ }^{17}$ La idea de comparar la esperanza de vida de Costa Rica, no sólo con los países de Centroamérica, sino también con los del Caribe, procede de un evaluador anónimo de este artículo a quien se reconoce y agradece esta aportación.
} 
complementan con los de CCP (2011), lo que permite extender el periodo de análisis y trabajar con series no suavizadas, a diferencia de las de BM (2011).

Como se aprecia en la Gráfica 4, la esperanza de vida máxima ha ido elevándose progresivamente durante el último siglo desde los 53,47 años de Noruega en 1900 hasta los 83,31 de Japón en 2009. ${ }^{18}$ La serie de Costa Rica se ha elevado, incluso, de forma más rápida, aprovechando el margen de mejora del que disponía, especialmente hasta finales de los setenta. En 1930, la expectativa de vida del país centroamericano aún era de 42,23 años, situándose a 22,73 de la de Australia. En 1940, la brecha ya se había reducido hasta los 19,81 años, en este caso respecto a Suecia, que con una cifra de 66,74 años marcaba el nuevo máximo mundial. Las décadas de los cuarenta y los cincuenta fueron las de mayor recuperación del terreno perdido. El crecimiento de la esperanza de vida a un ritmo de 1,08 años por cada año de calendario permitió que en 1950 la brecha se estrechara a 13,91 años, y en 1960 a tan sólo 8,43. De este modo, en apenas treinta años, la esperanza de vida de Costa Rica pasó de representar el 65\% del máximo alcanzable al $89 \%$. Una evolución que no hubiese sido posible sin el desarrollo de instituciones que ya se habían mostrado exitosas en otros países y que favorecieron la puesta en marcha de programas de salud, de seguridad social o de educación universal, reforzados por la ausencia de gasto militar tras la aprobación de la Constitución de 1949, que abolió las fuerzas armadas, y por la llegada de insecticidas, vacunas y medicamentos que redujeron la incidencia de las enfermedades de origen infeccioso y parasitario (Rosero-Bixby, 1991; Robles, 2006).

El proceso de convergencia, que podía haberse completado durante los sesenta, quedó interrumpido por los efectos de las erupciones del volcán Irazú entre 1963 y 1965. La elevada aridez de los piroclastos expulsados afectó gravemente a la fauna y flora del Valle Central, alterando las condiciones ecológicas preexistentes (Horton y McCaldin, 1964; Wille y Fuentes, 1975). En 1968, la erupción del volcán Arenal también causó importantes daños. En este caso, significó la completa devastación de un área de $15 \mathrm{~km}^{2}$, incluyendo los poblados de Pueblo Nuevo y Tabacón, y la afectación moderada de $230 \mathrm{~km}^{2}$ por la caída de las cenizas (Alvarado et al., 2006).

La reorganización del sector salud que tuvo lugar a principios de los setenta y que significó la transferencia de todos los hospitales del Ministerio de Salud Pública (MSP) a la Caja Costarricense de Seguridad Social (CCSS), que amplió su cobertura del 39\% al 70\% de la población entre 1970 y 1980 (Rosero-Bixby, 1994), pero especialmente la puesta en funcionamiento de programas de atención primaria en zonas rurales y barrios urbanos desfavorecidos (Haines y Avery, 1982; Rosero-Bixby, 1986 y 2004), facilitaron que la esperanza de vida de Costa Rica culminara su convergencia con la de los países más avanzados. La reducción de la tasa de mortalidad infantil desde 68,4\%o a 19,9\%o (CCP, 2011) ${ }^{19}$ que tuvo lugar

\footnotetext{
${ }^{18}$ Según datos de HMD (2011), la esperanza de vida máxima a lo largo del periodo 1900-2009 ha sido la marcada por las poblaciones de: Noruega (1900-2, 1904-6, 1911, 1920, 1926, 1946-53, 1955-6, 1958-9 y 1961), Suecia (1903, 1907-9, 1916, 1923, 1940-3, 1946 y 1963-74), Dinamarca (1910, 1912, 1914-5, 1918 y 1921), Islandia (1913, 1917, 1919, 1954, 1957-8, 1960, 1962 y 1975-80), Australia (1922, 1925, 1927, 1929-32 y 1944-5), Países Bajos (1924, 1928, 1933-9 y 1964) y Japón (1981-2009).

${ }^{19}$ Esta variación implica que la tasa de mortalidad infantil se redujo en un $70,89 \%$ en apenas diez años. La siguiente década con mayor contracción de la mortalidad infantil fue la de 1990-2000, cuando ésta se redujo "únicamente" un 31,65\%, desde 15,04\% a 10,28\%o. Horwitz (1987) encuentra que, entre 1970 y 1980, la reducción de la mortalidad infantil en Costa Rica fue del 69\%. Mayor aún fue el descenso de la tasa de mortalidad entre los menores de cinco años, que pasó de 19,61\% a 4,96\% entre 1970 y 1980, lo que supuso una reducción del 74,71\% de su valor inicial.
} 
durante esta década fue el principal mecanismo que ayudó a que la desventaja en esperanza de vida, que en 1970 era de 8,84 años, incluso más amplia que en 1960 (8,43 años), cayera hasta 1,03 en 1981, siendo esto lo más cerca que la población costarricense ha estado del máximo mundial desde el inicio de la transición demográfica.

A partir de entonces, en vez de proseguir el acercamiento secular que se ha descrito, se inició una fase de alejamiento progresivo respecto a la serie de máximos. La esperanza de vida de Costa Rica sólo se expandió 2,17 años entre 1981 y 2000, mientras que en Japón, el líder mundial, lo hizo en 4,74 años, más del doble, de forma que la distancia entre ambas series se amplió sucesivamente hasta los 2,03 años en 1990 y los 3,60 en 2000. Los problemas económicos por los que atravesó todo América Latina en los ochenta tuvieron incidencia en la sustancial reducción del gasto en salud pública que padeció Costa Rica en ese decenio (Rosero-Bixby, 1994:107), en el que la atención primaria en salud volvió a ser clave para explicar, en esta ocasión, el estancamiento de la esperanza de vida (Rosero-Bixby, 1995:2.6).

La reforma del sector salud iniciada en 1995, que primero se aplicó en las zonas menos densamente pobladas y de menor desarrollo socioeconómico (Rosero-Bixby, 2004), propició que la variable objeto de análisis reanudara su crecimiento a un ritmo semejante al de las naciones líderes, lo que no ha evitado que en 2009 la edad media de fallecimiento en Japón supere a la de Costa Rica en 3,93 años.

Como se observa en las Gráficas 2, 3 y 4, con independencia de las fuentes de datos utilizados, la esperanza de vida de Costa Rica creció a un ritmo acelerado hasta principios de los ochenta, cuando alcanzó su punto más cercano al máximo y ya no fue posible adoptar prácticas más saludables o incorporar técnicas que redujeran sensiblemente la mortalidad, dado que ésta ya era relativamente baja. A partir de entonces, la serie de esperanza de vida ha tendido a evolucionar en paralelo con la de máximos, esto es, incorporando los avances en la lucha contra la mortalidad al mismo ritmo que el líder mundial en este aspecto, Japón.

La Gráfica 2 muestra que este comportamiento de rápida convergencia y posterior evolución en paralelo no es exclusivo de Costa Rica. Las series de Belice y Panamá también presentan dinámicas semejantes, sólo que la distancia que mantienen respecto a la serie de mejores prácticas a partir de 1980 es superior. Según datos de BM (2011), Belice y Panamá se mantienen a una distancia del máximo de entre seis y siete años a partir de 1980. Costa Rica se sitúa alrededor de los tres años de diferencia, aproximadamente como ocurre en los casos de Cuba y Puerto Rico (Gráfica 3). Además, esta misma evolución ya ha sido descrita para el caso de España (Guijarro y Peláez, 2008 y 2009) y parece ser una situación generalizada ya que Oeppen y Vaupel (2002:1031) proponen proyectar la evolución futura de la esperanza de vida de cualquier población considerando que la brecha entre la serie de esa población en concreto y la de mejores prácticas tiende a permanecer más o menos estable en el tiempo. Por este motivo, cabe preguntarse si algún factor estructural puede estar limitando la capacidad de las distintas poblaciones para adoptar las mejores prácticas vigentes en cada momento, impidiendo que su

Respecto a las causas de esta evolución, Rosero-Bixby (1986) concluye que las mejoras en la atención primaria de la salud, incluyendo programas de nutrición, explican hasta un $41 \%$ del descenso, la atención intermedia explica el $32 \%$ (de modo que tres cuartas partes de la reducción de la mortalidad infantil son imputables a la acción de programas de salud pública), el progreso socioeconómico contribuye en un $22 \%$ y el descenso de la fertilidad en un $5 \%$. 
brecha respecto al máximo se cierre completamente y dando lugar a las trayectorias en paralelo detectadas.

Para analizar estas tendencias, se estima el modelo [1] descrito en el apartado 2, obteniendo un estadístico de Durbin-Watson igual a 0,6903 que lleva a rechazar la hipótesis de ausencia de autocorrelación. Debido a ello, se opta por estimar el modelo [3], de datos de panel con dinámica de errores, capaz, por tanto, de eludir el problema de la autocorrelación serial de los residuos.

Al estimar el modelo de la ecuación [3], se obtienen los siguientes resultados: ${ }^{20}$

$$
\begin{gathered}
\mathrm{y}_{\text {it }}^{*}=-\begin{array}{c}
0,084957 \\
(-0,2579)
\end{array} \mathrm{d}_{\text {lit }}^{*}-0,071736 \mathrm{~d}_{2 \mathrm{it}}^{*}+0,033887 \mathrm{~d}_{3 \mathrm{it}}^{*}-0,078384 \mathrm{~d}_{4 \mathrm{it}}^{*}+\mathrm{u}_{\mathrm{it}} \\
(-1,6322)
\end{gathered}
$$

Como se observa, solamente las diferencias en porcentajes de población urbana $\left(\mathrm{d}_{4 i t}\right)$ resultan ser plenamente significativas a la hora de explicar la distancia al máximo de las esperanzas de vida de los distintos países a lo largo del periodo 1995-2009. Dado el signo negativo del coeficiente y la especificación del modelo, mayores porcentajes de población urbana se asocian con menores brechas de esperanza de vida.

Ni las diferencias de ingreso $\left(\mathrm{d}_{1 \mathrm{it}}\right)$ ni de gasto público en educación $\left(\mathrm{d}_{3 \mathrm{it}}\right)$ resultan tener una incidencia estadísticamente significativa sobre los niveles relativos de esperanza de vida. De hecho, el coeficiente del gasto público en educación incluso presenta el signo contrario al que cabría esperar, lo que puede deberse a que el periodo contemplado es muy breve como para que la educación pueda tener efecto en la capacidad de supervivencia de la población.

La evidencia para el gasto público en salud $\left(\mathrm{d}_{2 \mathrm{it}}\right)$ no resulta del todo concluyente. Con un p-valor de 0,1036 queda en el límite del rechazo de su significatividad bajo un criterio de confianza del $90 \%{ }^{21}$ En cualquier caso, su signo es el que cabía esperar, indicando que mayores porcentajes de gasto público implican menores distancias de la esperanza de vida respecto al máximo.

\footnotetext{
${ }^{20}$ Para estimar los valores de los parámetros del modelo se utilizó el programa informático EViews 5.1. Entre paréntesis figuran los t-estadísticos asociados a cada parámetro estimado.

${ }^{21}$ Se comprobó que al prescindir de las dos variables claramente no significativas, los valores de los otros dos parámetros así como sus t-estadísticos apenas se modifican, lo que muestra la robustez de la estimación.
} 


\section{CONCLUSIONES}

En el presente artículo, se ha analizado no sólo la evolución de la esperanza de vida al nacimiento en Costa Rica a lo largo de casi un siglo de historia, sino también las dinámicas globales de esta variable. Ello ha permitido integrar las explicaciones particulares del caso de estudio en las tendencias mundiales, donde el margen para incorporar innovaciones relacionadas con la reducción de la mortalidad resulta un factor determinante.

A nivel global, se ha confirmado el proceso de convergencia al alza de las esperanzas de vida advertido por Wilson (2001), para lo cual se adaptaron los conceptos así como las técnicas de cálculo de las convergencias $\beta$ y $\sigma$ descritas por Barro y Sala-i-Martin (1990, 1991, 1992 y 1995) para variables de tipo económico. Concretamente, se encontró que la esperanza de vida al nacimiento aumentó más en las poblaciones que en 1960 presentaban valores más bajos, incrementándose 0,32 años adicionales entre 1960 y 2009 por cada año de rezago inicial. Las décadas de los sesenta y los setenta, así como durante el nuevo milenio, fueron los periodos de convergencia más intensa. Desde mediados de los ochenta y en los noventa, la convergencia se interrumpió debido al mal desempeño de los países del África no mediterránea. Este proceso de convergencia de las series de esperanza de vida también se observa en el interior de la región centroamericana, donde Costa Rica ha sido referente a lo largo del último medio siglo.

Al situar la serie de esperanza de vida de la población costarricense en el contexto global, se advirtió que ésta fue aproximándose a la de mejores prácticas a un ritmo superior a la media hasta principios de la década de los ochenta. En 1981 se situó a tan sólo 1,03 años del máximo mundial. Desde entonces, dado el escaso margen ya existente para incorporar innovaciones en favor de la salud, la serie tendió a estancarse y luego a evolucionar en paralelo respecto a la de Japón, el líder en este aspecto.

Entre los factores estructurales que pueden estar impidiendo la completa convergencia con el máximo de las series de esperanza de vida, no sólo de Costa Rica, sino también de otras poblaciones que se encuentran en una situación similar, se detectó que el porcentaje de producto interno bruto destinado a gasto público en salud y, especialmente, el grado de urbanización son variables significativas. No se tuvo evidencia de que el ingreso nacional bruto per cápita, ni el gasto público en educación tuvieran incidencia en este sentido. Cabe destacar que no se pudo obtener información suficiente para verificar si la pobreza o la desigualdad en la distribución de ingresos y renta ayudan a explicar los diferentes niveles de esperanza de vida. Futuras investigaciones deberán abordar esta cuestión.

El caso de Costa Rica, con un ingreso nacional bruto per cápita que ni siquiera alcanza a representar un tercio del de Japón (BM, 2011), ilustra el hecho de que el nivel económico medio de los países no es tan relevante para conseguir altos niveles de esperanza de vida. La adecuación e implementación de medidas que garanticen la mayor difusión posible de los medios de salud entre la población parecen ser aspectos más importantes a la vista de los resultados obtenidos en esta investigación. 


\section{BIBLIOGRAFÍA}

Alvarado, G.E., Soto, G.J., Schmincke, H.U., Bolge, L.L., \& Sumita, M. (2006). The 1968 andesitic lateral blast eruption at Arenal volcano, Costa Rica. Journal of Volcanology and Geothermal Research, 157(1-3), 9-33.

Anand, S., \& Ravallion, M. (1993). Human development in poor countries: on the role of private incomes and public services. The Journal of Economic Perspectives, 7(1), 133-150.

Arriaga, E.E. (1968). New life tables for Latin American populations in the nineteenth and twentieth centuries. Berkeley: University of California Press.

Arriaga, E.E., \& Davis, K. (1969). The pattern of mortality change in Latin America. Demography, 6(3), 223-242.

Bagnall, R.S. (1993). Egypt in late antiquity. Princeton: Princeton University Press.

Barro, R.J., \& Sala-i-Martin, X. (1990). Economic growth and convergence across the United States. NBER Working Paper Series, 3419. Cambridge: National Bureau of Economic Research.

Barro, R.J., \& Sala-i-Martin, X. (1991). Convergence across states and regions. Brookings Papers on Economic Activity, 22(1), 107-182.

Barro, R.J., \& Sala-i-Martin, X. (1992). Convergence. Journal of Political Economy, 100(2), 223251 .

Barro, R.J., \& Sala-i-Martin, X. (1995). Economic growth. Nueva York: McGraw-Hill.

Benítez, R., \& Cabrera, G. (1967). Tablas abreviadas de mortalidad de la población de México, 1930, 1940, 1950, 1960. México, D.F.: El Colegio de México.

Bhargava, A., Franzini, L., \& Narendranathan, W. (1982). Serial correlation and the fixed effects model. Review of Economic Studies, 49(4), 533-549.

BM (2011). Datos: indicadores. Washington, D.C.: Banco Mundial. Consulta del 28 de octubre, 2011, de http://datos.bancomundial.org/indicador.

Bongaarts, J. (2006). How long will we live? Policy Research Division Working Paper, 215. Nueva York: The Population Council.

Bourgeois-Pichat, J. (1952). Essai sur la mortalité 'biologique' de l'homme. Population, 7(3), 381-394. 
Camposortega, S. (1992). Análisis demográfico de la mortalidad en México 1940-1980. México, D.F.: El Colegio de México.

CCP (2011). Indicadores demográficos: esperanza de vida 1930-. San José: Centro Centroamericano de Población. Consulta del 21 de noviembre, 2011, de http://ccp.ucr.ac.cr/observa/CRindicadores/evida.html.

Chenery, H.B., \& Syrquin, M. (1975). Patterns of development 1950-1970. Londres: Oxford University Press.

Corona, R., Jiménez, R., \& Minujín, A. (1982). La mortalidad en México. México, D.F.: Instituto de Investigaciones Sociales de la UNAM.

Davis, K. (1956). The amazing decline of mortality in underdeveloped areas. The American Economic Review, 46(2), 305-318.

Frejka, T. (1981). Long-term prospects for world population growth. Population and Development Review, 7(3), 489-511.

Guijarro, M., \& Peláez, Ó. (2008). La longevidad globalizada: un análisis de la esperanza de vida en España (1900-2050). Scripta Nova, 12(260). Consulta del 21 de noviembre, 2011, de http://www.ub.edu/geocrit/sn/sn-260.htm.

Guijarro, M., \& Peláez, Ó. (2009). Proyección a largo plazo de la esperanza de vida en España. Estadística Española, 51(170), 193-220.

Haines, M.R., \& Avery, R.C. (1982). Differential infant and child mortality in Costa Rica: 19681973. Population Studies, 36(1), 31-43.

HMD (2011). Human Mortality Database. Berkeley: University of California; \& Rostock: Max Planck Institute for Demographic Research. Consulta del 28 de octubre, 2011, de http://www.mortality.org o http://www.humanmortality.de.

Horton, R.J.M., \& McCaldin, R.O. (1964). Observations on air pollution espects of Irazú volcano, Costa Rica. Public Health Reports, 79, 925-929.

Horwitz, A. (1987). Comparative public health: Costa Rica, Cuba, and Chile. Food and Nutrition Bulletin, 9(3), 19-29.

Livi-Bacci, M. (1978). La fecundidad y el crecimiento demográfico en España en los siglos XVIII y XIX. En Glass, D.V., \& Revelle, R. (dir.). Población y cambio social: estudios de demografía histórica. 176-187. Madrid: Tecnos.

Livi-Bacci, M. (2002). Historia mínima de la población mundial. Barcelona: Ariel.

Manton, K.G., Stallard, E., \& Tolley, H.D. (1991). Limits to human life expectancy: evidence, prospects, and implications. Population and Development Review, 17(4), 603-637. 
Oeppen, J., \& Vaupel, J.W. (2002). Broken limits to life expectancy. Science, 296(5570), 10291031.

Olshansky, S.J., \& Ault, A.B. (1986). The fourth stage of epidemiologic transition: the age of delayed degenerative diseases. The Milbank Memorial Fund Quarterly, 64(3), 355-391.

Omran, A.R. (1971). The epidemiologic transition. The Milbank Memorial Fund Quarterly, 49(4), 509-538.

Pérez-Brignoli, H. (2010). América Latina en la transición demográfica, 1800-1980. Población y Salud en Mesoamérica, 7(2), artículo 1a. Consulta del 28 de febrero, 2012, de http://ccp.ucr.ac.cr/revista/volumenes/7/7-2/7-2-1a/index.htm.

PNUD (2006). Informe sobre desarrollo humano 2006. Más allá de la escasez: poder, pobreza y la crisis mundial del agua. Nueva York: Programa de las Naciones Unidas para el Desarrollo.

Preston, S.H. (1975). The changing relation between mortality and level of economic development. Population Studies, 29(2), 231-248.

Riley, J.C. (2001). Rising life expectancy: a global history. Nueva York: Cambridge University Press.

Robles, A. (2006). Cambio demográfico, inversión social y diferencias generacionales en Costa Rica. Notas de Población, 82, 69-94.

Rodgers, G.B. (1979). Income and inequality as determinants of mortality: an international crosssection analysis. Population Studies, 33(2), 343-351.

Rosero-Bixby, L. (1986). Infant mortality in Costa Rica: explaining the recent decline. Studies in Family Planning, 17(2), 57-65.

Rosero-Bixby, L. (1991). Socioeconomic development, health interventions and mortality decline in Costa Rica. Scandinavian Journal of Social Medicine. Supplementum, 46, 33-42.

Rosero-Bixby, L. (1994). La disminución de la mortalidad de adultos en Costa Rica. Notas de Población, 60, 103-139.

Rosero-Bixby, L. (1995). La mortalidad infantil en Costa Rica 1981-1990. En Gómez, V.M. (ed.). Actualidad demográfica de Costa Rica 1994. 2.1-2.12. San José: Fondo de Población de las Naciones Unidas.

Rosero-Bixby, L. (2004). Evaluación del impacto de la reforma del sector de la salud en Costa Rica mediante un estudio cuasiexperimental. Revista Panamericana de Salud Pública, 15(2), 94-103. 
Spengler, J.J. (1978). Los factores demográficos y el primer desarrollo económico moderno. En Glass, D.V., \& Revelle, R. (dir.). Población y cambio social: estudios de demografía histórica. 96-107. Madrid: Tecnos.

Vallin, J. (1995). La demografía. Madrid: Alianza.

Whelpton, P.K., Eldridge, H.T., \& Siegel, J.S. (1947). Forecasts of the population of the United States 1945-1975. Washington, D.C.: U.S. Bureau of the Census.

Wille, Á., \& Fuentes, G. (1975). Effects of the Irazu volcano (Costa Rica) on various insects. Revista de Biología Tropical, 23(2), 165-175.

Wilson, C. (2001). On the scale of global demographic convergence 1950-2000. Population and Development Review, 27(1), 155-171. 
Tabla 1. Coeficientes de convergencia $\beta$ para la esperanza de vida al nacimiento, 1960-2009

\begin{tabular}{|c|c|c|c|c|c|c|}
\hline \multicolumn{7}{|c|}{152 poblaciones } \\
\hline & $1960-09$ & $1960-70$ & $1970-80$ & $1980-90$ & $1990-00$ & $2000-09$ \\
\hline$\beta$ & $-0,3274$ & $-0,0862$ & $-0,0934$ & $-0,0530$ & $-0,0256$ & $-0,0695$ \\
\hline p-valor & 0,0000 & 0,0000 & 0,0000 & 0,0287 & 0,3703 & 0,0000 \\
\hline $\mathrm{r}^{2}$ & 0,2962 & 0,1690 & 0,1620 & 0,0316 & 0,0052 & 0,1892 \\
\hline \multicolumn{7}{|c|}{ Sin África no mediterránea } \\
\hline & $1960-09$ & $1960-70$ & $1970-80$ & $1980-90$ & $1990-00$ & $2000-09$ \\
\hline$\beta$ & $-0,6108$ & $-0,1581$ & $-0,1403$ & $-0,2362$ & $-0,1408$ & $-0,0768$ \\
\hline p-valor & 0,0000 & 0,0000 & 0,0000 & 0,0000 & 0,0000 & 0,0000 \\
\hline $\mathrm{r}^{2}$ & 0,7266 & 0,3284 & 0,2088 & 0,4644 & 0,1887 & 0,1540 \\
\hline \multicolumn{7}{|c|}{ Centroamérica } \\
\hline & $1960-09$ & $1960-70$ & $1970-80$ & $1980-90$ & 1990-00 & 2000-09 \\
\hline$\beta$ & $-0,6636$ & $-0,0913$ & $-0,0200$ & $-0,3070$ & $-0,3232$ & $-0,1723$ \\
\hline p-valor & 0,0008 & 0,0111 & 0,8903 & 0,0167 & 0,0035 & 0,0783 \\
\hline$r^{2}$ & 0,9138 & 0,7555 & 0,0042 & 0,7137 & 0,8436 & 0,4937 \\
\hline
\end{tabular}

Fuente: Elaboración propia con datos de BM (2011).

Gráfica 1. Desviación típica de las esperanzas de vida al nacimiento, 1960-2009 (Convergencia $\sigma)$

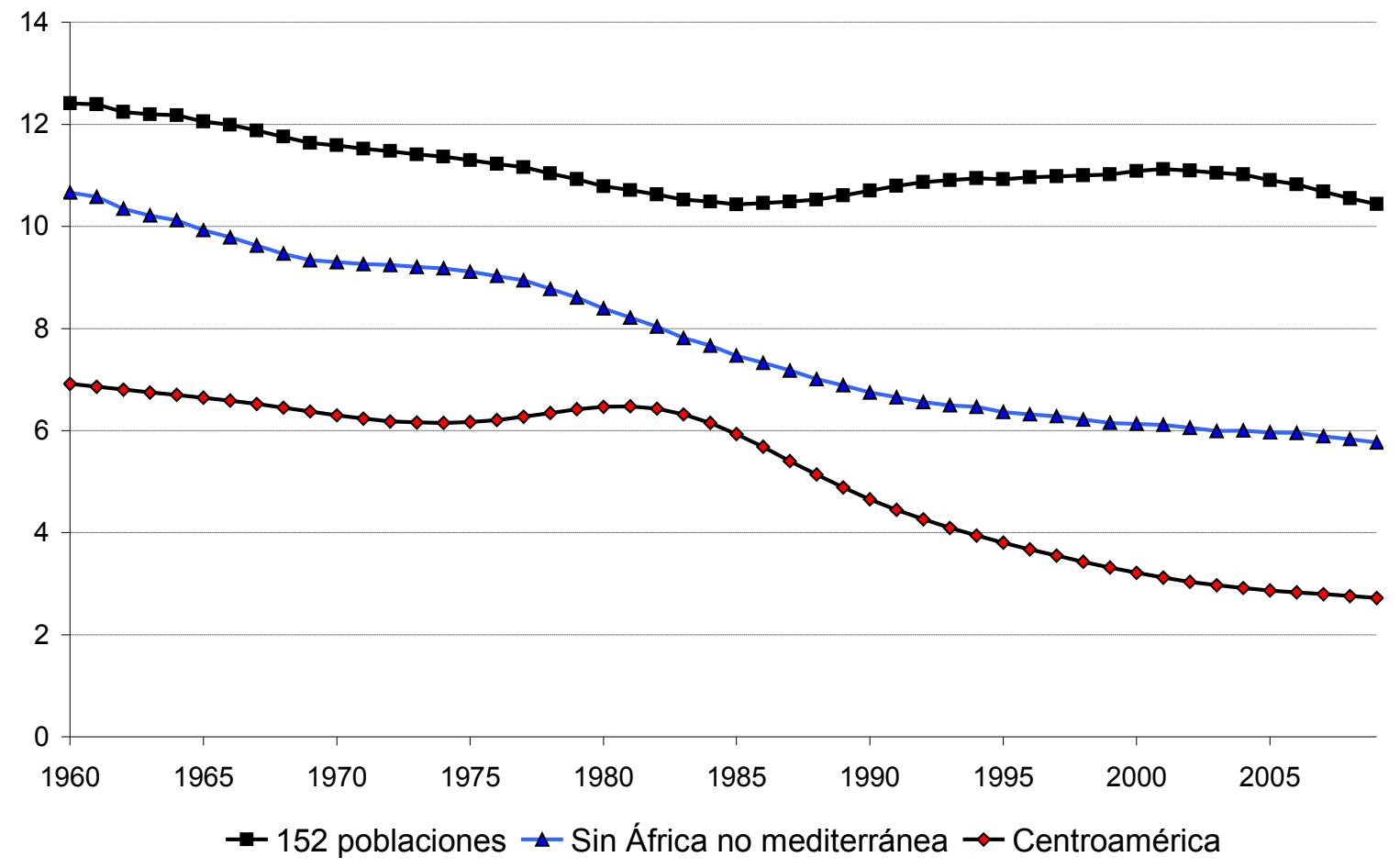

Fuente: Elaboración propia con datos de BM (2011). 
Gráfica 2. Distancia en años de las esperanzas de vida de los países de Centroamérica respecto al máximo mundial, 1960-2009

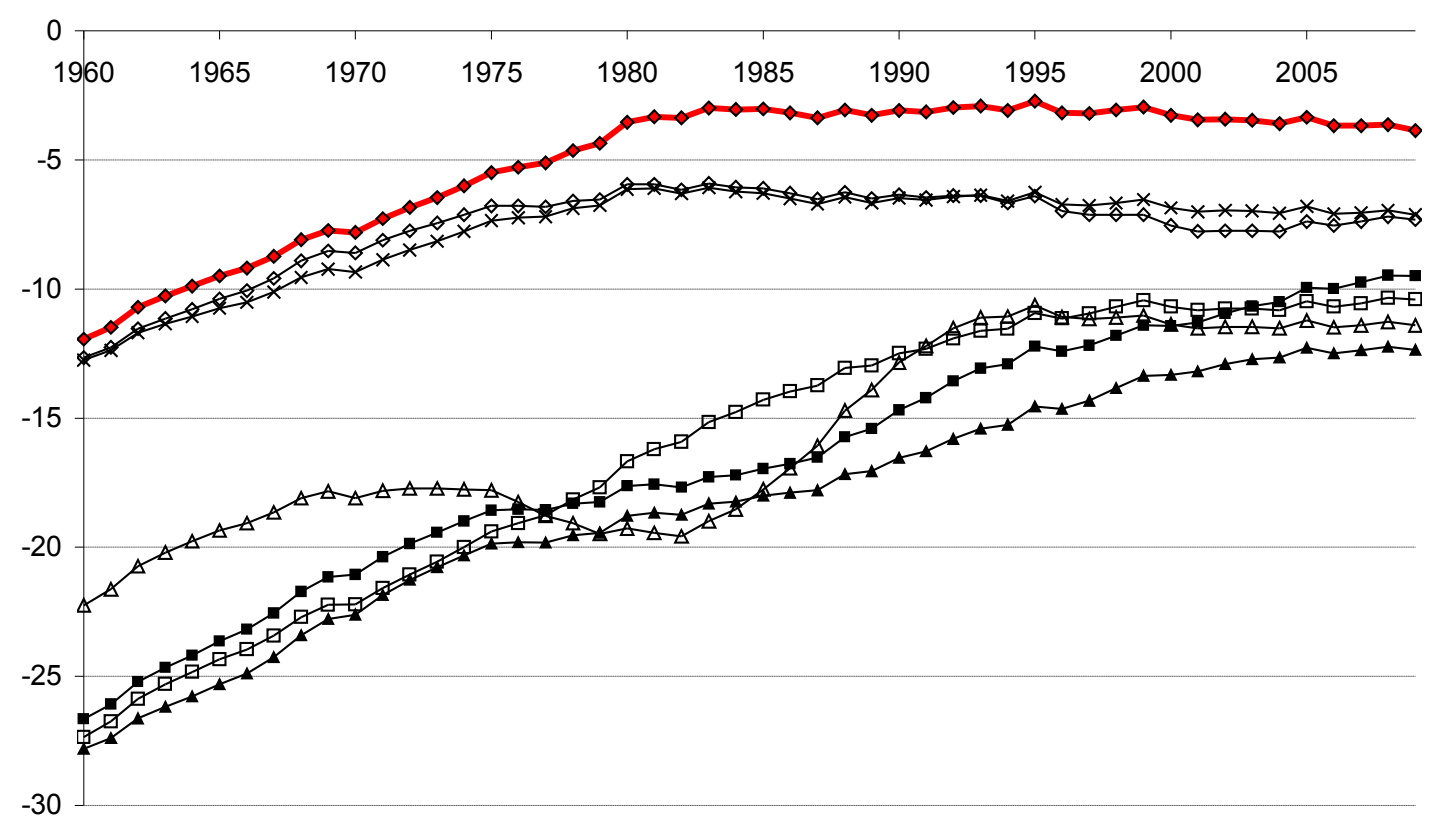

$\neg$ Belice $\leadsto$ Costa Rica $\triangle$ El Salvador $\rightarrow$ Guatemala $\rightarrow$ Honduras $\rightarrow$ Nicaragua $\star$ Panamá Fuente: Elaboración propia con datos de BM (2011).

Gráfica 3. Distancia en años de las esperanzas de vida de Costa Rica y los principales países del Caribe respecto al máximo mundial, 1960-2009

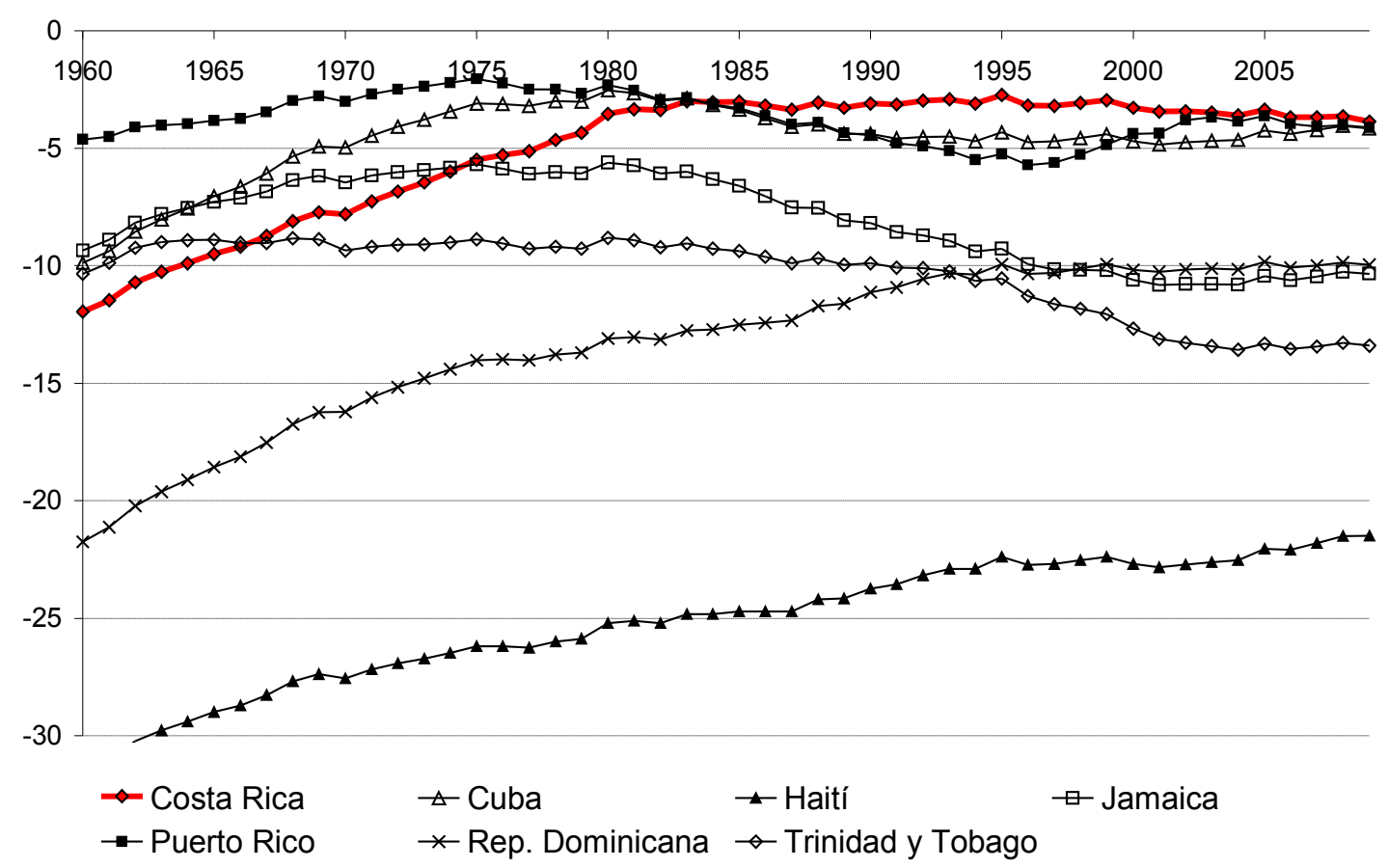

Fuente: Elaboración propia con datos de BM (2011). 
Gráfica 4. Evolución a largo plazo de la esperanza de vida al nacimiento de Costa Rica en comparación con otras poblaciones

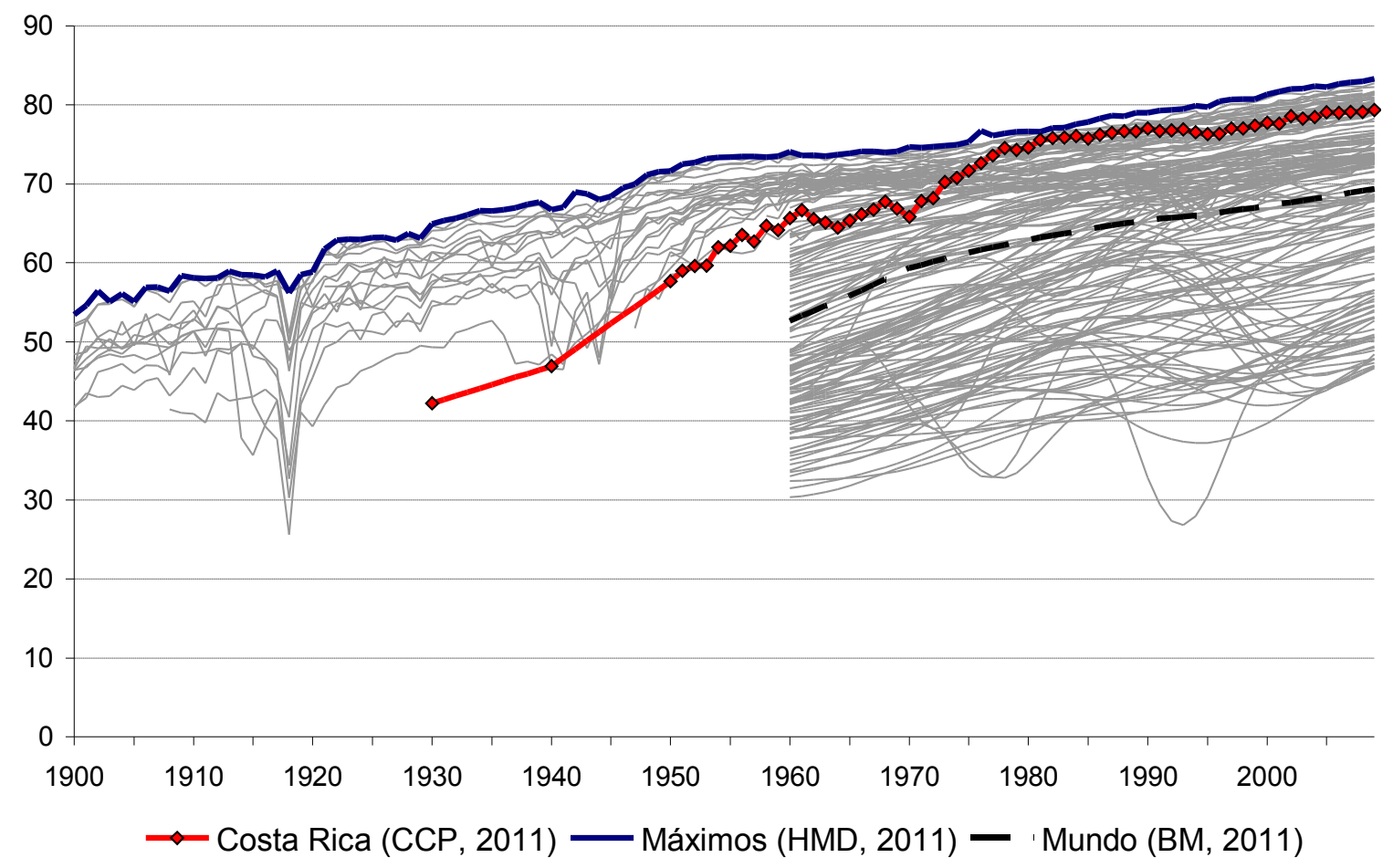

Fuente: Elaboración propia con datos de BM (2011), CCP (2011) y HMD (2011). 